\title{
The microbiota knows: handling-stress and diet transform the microbial landscape in the gut content of rainbow trout in RAS
}

\section{Marvin Suhr}

Institute of Animal Nutrition and Physiology, Christian-Albrechts-University Kiel

Finn-Thorbjörn Fichtner-Grabowski

Institute of Animal Nutrition and Physiology, Christian-Albrechts-University Kiel

Henrike Seibel

Fraunhofer Research Institution for Individualized and Cell-Based Medical Engineering (IMTE)

Corinna Bang

Institute of Clinical Molecular Biology, Christian-Albrechts-University Kiel

\section{Andre Franke}

Institute of Clinical Molecular Biology, Christian-Albrechts-University Kiel

\section{Carsten Schulz}

Fraunhofer Research Institution for Individualized and Cell-Based Medical Engineering (IMTE)

Stéphanie Céline Hornburg ( $\nabla$ hornburg@aninut.uni-kiel.de )

Institute of Animal Nutrition and Physiology, Christian-Albrechts-University Kiel

\section{Research Article}

Keywords: intestinal microbiome, stress, plant-based diets, aquaculture, Oncorhynchus mykiss, 16S rRNA gene

Posted Date: February 21st, 2022

DOI: https://doi.org/10.21203/rs.3.rs-1369558/v1

License: (c) (1) This work is licensed under a Creative Commons Attribution 4.0 International License. Read Full License 


\section{Abstract}

Background: The aim of the present study was to characterize the effects of handling stress on the microbiome in the intestinal gut contents of rainbow trout (Oncorhynchus mykiss) from two different breeding lines fed with plant-based diets. Two experimental diets were provided for 59 days to all female trout and half of the fish were shooed with a fishing net twice per day to induce long-term stress (groups 0 and 1 ) in a fully recirculating aquaculture system (RAS). Experimental diets were formulated according to commercial trout diets differing in fish meal (35\% diet $F, 7 \%$ diet $V)$ and plant-based proteins ( $47 \%$ diet F, 73\% diet V).

Results: No differences in performance parameters were found between treatment groups. By using $16 \mathrm{~S}$ rRNA amplicon sequencing of the hypervariable region V3/V4 we examined the microbial community in the total gut content of fish at the end of the trial. We discovered no significant differences in alpha diversity induced by one of the two factors within both trout lines. However, the microbial composition is significantly driven by an interaction of stress and diet in trout line A. Otherwise, in trout line B the main factor was stress. The communities of both breeding lines were predominantly colonized with bacteria from the phyla Fusobacteriota, Firmicutes, Proteobacteria, Actinobacteriota and Bacteroidota. The most varying and most abundant taxa have been found to be Firmicutes and Fusobacteriota, where at the genus level Cetobacterium and Mycoplasma are key components in terms of adaptation.

Conclusion: We conclude, that microbial gut composition is highly influenced by stress which is presupposed by diet formulation and trout origin but constant microbial diversity and performance parameter.

\section{Introduction}

The global amount of fish produced in aquaculture has been constantly growing over the past twenty years, as did the consumer's demand [1]. This food producing sector is a fundamental element in providing human population with high-quality protein. As in most animal farming systems, however, consumers demand to a greater extent for high standards regarding animal welfare and nutrition, as well as sustainable production procedures. Despite aquaculture being a major and important pillar in food production, it lacks for sustainability when it comes to feeding fish meal and fish oil made up from marine fish stocks which suffer from serious depletion in the last decades [1]. Therefore, it is necessary that alternative protein sources with appropriate nutritious qualities are on demand. Many years of research found suitable substitutes, such as oilseeds, legumes and cereal grains [2,3] or insect meal [4]. Compelling quality measures for alternative feeds are low percentages of starch, fiber and antinutrients [5]. Nevertheless, it is well established, that dietary components change environmental parameters for bacteria residing within the intestine of animals, the so-called microbiota [6, 7] who earn an essential role in digestion, nutrient absorption, immune function, protection against pathogens or modulation as part of the gut-brain-axis [8]. Hence, diet formulation and origin are able to alter the composition of the intestinal microbiota $[7,9]$ and thus the health condition of the host. Desai and colleagues revealed, that a plant- 
based diet increased the overall richness, diversity and especially the abundance of lactic acid bacteria (LAB) from the phylum Firmicutes [10]. Lactococcus has been used as probiotics on the basis of its inhibitory effects against fish pathogens shown in rainbow trout [11] and Atlantic salmon [12] [13]. It has also been proposed, that health beneficial commensals Streptococcus, Leuconostoc and Weissella seem to be promoted, when fish fed a diet containing rape seed and pea meal. Additionally, juvenile rainbow trout are less susceptible to infection of Yersinia ruckeri diets and thus referring to a prebiotic effect of plant-derived products [14]. The effects of plant-based fish feed on the intestinal microbiome, and thus host health, are diverse and need to be evaluated when it comes to fish welfare in a stressful environment.

Then again and coherent, lipase and protease-producing bacteria are associated with a carnivorous diet but the overall diversity compared to herbivorous fish decreases [15]. However, a constant set of bacteria seems to sustain and form a core microbiota in several species [16-18] inter alia rainbow trout [3]. As several studies support, the core microbiota of rainbow trout is dominated by phyla Bacteroidetes, Proteobacteria, Firmicutes and Actinobacteria which seems to be consistent in wild-caught and selectively bred fish. Eventually, a well-balanced microbiome inhabits beneficial members which provide the host with valuable extracellular enzymes (carbohydrates, cellulases, phosphatases, esterases, lipases, proteases), fatty acids, vitamins and amino acids [19].

Besides an optimal nutrient supply, rearing conditions preferably free of handling, group or environmental stress is desired for an effective aquaculture. Handling stress, e.g. transportation, sorting, grading or rearing conditions such as temperature $[20]$ and stocking rates $[3,21]$ are common stress factors. Possible consequences are, e.g. an increased susceptibility towards pathogenic diseases [22]. Previous studies on zebrafish pointed to a connection between stress resistance and the microbiome [23] and studies on rodents revealed a severe change in gut microbiome induced by stress [24, 25]. Further, in mammals a negative impact on immunity and microbiome disruption induced by stress was reported which may cause e.g. neurological disease [8] and in wild and cultured fish stress is a trigger for increasing susceptibility to disease. Studies have been conducted on the negative impact of stress on the microbiome mediated via the stress hormone cortisol and resulted in individual changes in taxa [26]. An important role of the intestinal microbiome is being a regulator of the gut-brain-axis in vertebrates which is acting in the sympathetic and parasympathetic as well as central nerval system. Considering, that microbial colonization is influenced by the diet type, it can be hypothesized that stress response is affected as well. Conversely, as mentioned previously, stress may disrupt microbial gut composition and thus an interaction between those two factors has to be considered.

Thus, this study aimed to examine the following hypotheses: (i) the intestinal microbiome of rainbow trout is affected by an external handling stressor and (ii) the inclusion of plant-based protein sources in rainbow trout diets alters the effect of handling stress on the microbial community. In order to evaluate possible genetical influences on this interaction two different rainbow trout breeding lines were examined.

\section{Results}




\section{Rainbow trout performance}

At the end of the trial, individual final body weights (FBW) of sampled fish $(n=240)$ did not differ significantly between treatments. Performance parameters were calculated based on group weights from day 50 and are shown in Table 1: neither stress nor diet had a significant influence on performance parameters, except for a statistically increased daily feed intake (DFI) in trout from both breeding lines fed with diet $V$ in comparison to fish fed with $\operatorname{diet} F(A: p=9.78 e-06 ; B: p=0.0085)$.

Table 1

Rainbow trout performance values and read counts of experimental treatments. Mean value $\pm S D, n=3$.

\begin{tabular}{|c|c|c|c|c|c|c|c|}
\hline Treatment & reads & FBW $($ IBW*) & SGR & FCR & DFI & PER & PRE \\
\hline A-init & $\begin{array}{l}19,293 \pm \\
9,110\end{array}$ & $\begin{array}{l}132.14 \pm \\
15.55^{\star}\end{array}$ & & & & & \\
\hline$A-0-V$ & $\begin{array}{l}23,090 \pm \\
17,742\end{array}$ & $\begin{array}{l}347.804 \pm \\
38.25\end{array}$ & $\begin{array}{l}1.76 \pm \\
0.03\end{array}$ & $\begin{array}{l}0.88 \pm \\
0.01\end{array}$ & $\begin{array}{l}1.54 \pm \\
0.00\end{array}$ & $\begin{array}{l}2.73 \pm \\
0.04\end{array}$ & $\begin{array}{l}44.32 \pm \\
0.92\end{array}$ \\
\hline$A-1-V$ & $\begin{array}{l}13,451 \pm \\
9,380\end{array}$ & $\begin{array}{l}343.013 \pm \\
45.00\end{array}$ & $\begin{array}{l}1.73 \pm \\
0.06\end{array}$ & $\begin{array}{l}0.89 \pm \\
0.03\end{array}$ & $\begin{array}{l}1.54 \pm \\
0.01\end{array}$ & $\begin{array}{l}2.69 \pm \\
0.10\end{array}$ & $\begin{array}{l}43.99 \pm \\
5.98\end{array}$ \\
\hline$A-0-F$ & $\begin{array}{l}15,996 \pm \\
12,833\end{array}$ & $\begin{array}{l}359.82 \pm \\
34.10\end{array}$ & $\begin{array}{l}1.78 \pm \\
0.02\end{array}$ & $\begin{array}{l}0.85 \pm \\
0.01\end{array}$ & $\begin{array}{l}1.51 \pm \\
0.01\end{array}$ & $\begin{array}{l}2.77 \pm \\
0.02\end{array}$ & $\begin{array}{l}45.79 \pm \\
0.10\end{array}$ \\
\hline$A-1-F$ & $\begin{array}{l}2,390 \pm \\
3,653\end{array}$ & $\begin{array}{l}350.088 \pm \\
40.50\end{array}$ & $\begin{array}{l}1.72 \pm \\
0.03\end{array}$ & $\begin{array}{l}0.87 \pm \\
0.01\end{array}$ & $\begin{array}{l}1.49 \pm \\
0.01\end{array}$ & $\begin{array}{l}2.70 \pm \\
0.03\end{array}$ & $\begin{array}{l}41.49 \pm \\
3.48\end{array}$ \\
\hline B-init & $\begin{array}{l}21,930 \pm \\
13,531\end{array}$ & $\begin{array}{l}149.74 \pm \\
18.05^{\star}\end{array}$ & & & & & \\
\hline$B-0-V$ & $\begin{array}{l}4,824 \pm \\
9,864\end{array}$ & $\begin{array}{l}433.00 \pm \\
58.23\end{array}$ & $\begin{array}{l}1.74 \pm \\
0.04\end{array}$ & $\begin{array}{l}0.89 \pm \\
0.02\end{array}$ & $\begin{array}{l}1.54 \pm \\
0.03\end{array}$ & $\begin{array}{l}2.71 \pm \\
0.07\end{array}$ & $\begin{array}{l}41.19 \pm \\
0.42\end{array}$ \\
\hline B-1-V & $\begin{array}{l}21,880 \pm \\
17,287\end{array}$ & $\begin{array}{l}421.73 \pm \\
69.35\end{array}$ & $\begin{array}{l}1.76 \pm \\
0.07\end{array}$ & $\begin{array}{l}0.87 \pm \\
0.03\end{array}$ & $\begin{array}{l}1.53 \pm \\
0.01\end{array}$ & $\begin{array}{l}2.76 \pm \\
0.10\end{array}$ & $\begin{array}{l}39.40 \pm \\
6.48\end{array}$ \\
\hline B-0-F & $4,346 \pm 867$ & $\begin{array}{l}410.63 \pm \\
43.93\end{array}$ & $\begin{array}{l}1.75 \pm \\
0.05\end{array}$ & $\begin{array}{l}0.85 \pm \\
0.02\end{array}$ & $\begin{array}{l}1.49 \pm \\
0.01\end{array}$ & $\begin{array}{l}2.75 \pm \\
0.07\end{array}$ & $\begin{array}{l}45.48 \pm \\
0.90\end{array}$ \\
\hline B-1-F & $\begin{array}{l}31,635 \pm \\
17,212\end{array}$ & $\begin{array}{l}415.29 \pm \\
56.39\end{array}$ & $\begin{array}{l}1.78 \pm \\
0.02\end{array}$ & $\begin{array}{l}0.85 \pm \\
0.01\end{array}$ & $\begin{array}{l}1.51 \pm \\
0.00\end{array}$ & $\begin{array}{l}2.77 \pm \\
0.04\end{array}$ & $\begin{array}{l}44.55 \pm \\
0.91\end{array}$ \\
\hline $\begin{array}{l}\text { total no. } \\
\text { Reads }\end{array}$ & $2,446,407$ & & & & & & \\
\hline
\end{tabular}

FBW final body weight (g), IBW initial body weight (g), SGR specific growth rate, FCR feed conversion ratio, $D F I$ daily feed intake, $P E R$ protein efficiency ratio, $P R E$ protein retention efficiency 
To assess the intestinal microbial composition of individual trout, bacterial DNA extracted from total gut content at the 16S rRNA V3-V4 region was sequenced. Demultiplexed Illumina MiSeq reads comprised of 2x300 bp were imported in Qiime2 with a raw total read count of 7,458,158 per read direction. After primer trimming and denoising, 5,408,391 reads which correspond to a total loss of $27.48 \%$ reads, were left for further analysis. After taxonomic classification, ASVs identified as cyanobacteria or mitochondrial origin, were removed from the dataset as they are considered as contamination. Samples from batches with contaminated negative controls, i.e. with reads originating from fish intestinal microbes, where removed from further analysis. Generally, samples were considered as contaminated, when extraction control from that run had a considerable high number of reads. In total, 145 samples were left for further analysis, composed of 41 initial samples and 104 feces samples. For the experimental dataset (without RAS samples) a total ASV count of 764 (69 samples) for group A and 540 (35 samples) for group B were left for further analysis. Finally, 2,446,407 reads remained for downstream analysis including initial sampling ( $A: n=21, B: n=20$ ) and the experimental samples. The average number of reads is 16,872 .

\section{The impact of Diet and Stress on Microbial Diversity}

Alpha diversity measures including observed ASVs (richness) as a qualitative, Shannon Diversity index and Simpson index as a quantitative parameter, were estimated based on genus level data to compare taxonomic diversity between treatments. Significant differences in observed ASVs could be accounted for trout from line $B$, fed with diet $F$ which were either stressed or not $(p=0.00542$, Fig. $1 A)$. Evaluation of trout line $A$ revealed no significant interaction or any impact of diet or stress on diversity measures. The average observed ASVs across all treatments within each trout line, did not vary significantly between $A$ $(\sim 28)$ and $B(\sim 29)$. Observed ASVs from initial sampling is significantly increased in trout line $A(p=$ 1.56e-06, data not shown) compared to the average of all experimental treatments. In trout line $B$ no such effect was observed. Considering richness and evenness (Shannon) in treatments from trout line $A$, maximum mean diversity was obtained in treatment $0-\mathrm{F}$ and $0-\mathrm{V}$. Less diversity was observed in A-1-F and $A-1-V$ though not significantly. Consistent with the previous parameter, an inversed impact of fish from line $B$ can be registered, where stress is associated with increased Shannon diversity. Shannon and Simpson index in trout line B were significantly higher prior to the trial start $(p=0.001$, Figure $S 2$, while in trout line A no differences in sampling time were observed.

\section{Microbial composition is affected by stress and diet}

First, we intended to determine overall microbial community composition altered by diet-by-stress treatments in trout lines. For Nonmetric Multidimensional Scaling (NMDS) we calculated unweighted UniFrac distances to evalute community structure by integrating phylogenetic information and presence/absence information and further weighted UniFrac distances which additionally includes abundance data based on genus-level data. First, a model which inclues only intial samples from $A$ and $B$, demonstrates significant differences between both trout lines prior the trial $(p=0.001,0.08063$, PERMANOVA, Table T2). Samples prior the experiment and samples post treatment within each trout line 
show significant separation ( $A p=0.001, R^{2}=0.065 ; B p=0.001, R^{2}=0.075$, PERMANONA; Fig. 2) based on unweighted UniFrac distances and by mild clustering in NMDS.

Testing for differences in a model using all of the previosly mentioned data (A, B, A-init, B-init; Figure S5) shows, that at the end of the experiment samples from trout line $A$ and $B$ are not significantly different from each other $(p=0.183)$. According to the statistic above, significant differences between the initial sampling from both trout lines ( $A$-init $-B$-init, $p=0.004)$ and within each trout line $(A-A$-init, $p=0.004 ; B$ - B-init, $p=0.004$ ) could be accounted.

Graphical representation of unweighted UniFrac of trout line A (Fig. 3A) revealed no clustering by treatment but rather if the fish were stessed or not. However, PERMANOVA results revealed significant differences in microbial composition among treatments explained by the interaction factor stress and diet $(p=0.034)$. This was also confirmed by weighted UniFrac $(p=0.017$; Fig. $3 B)$. Results of pairwise statistical comparison indicated scarce significant differences between $0-\mathrm{V}: 1-\mathrm{V}(p=0.055)$ in unweighted UniFrac, but significant differences in stressed trouts fed either diet $F$ or $V(p=0.046)$ in weighted UniFrac. Additionally, differences were also observed in treatments fed diet $F$ where the stress factor had a significant impact $(p=0.028)$.

Individuals from line $B$ significantly differ by factor stress (unweighted: $p=0.001$; weighted: $p=0.046$; PERMANOVA) which is also confirmed by NMDS visualization (Fig. 3B and D). However, no interaction between diet and stress was observed in trout line B. The variablity of microbial communities within groups was further tested by dispersion analysis incorporating both UniFrac distances on genus-level data, respectively. Congruent with previous results, significant increased values for dispersion (distance to centroid for each sample in associated group) in trout line B for stressed fish across diet was detected in unweighted UniFrac distance (ANOVA, $p=0.041$ ). No significant differences in trout line A was observed.

\section{Taxonomical composition of microbial communities}

To further analyze the microbial gut composition of rainbow trout and identify taxa which provoke the differences mentioned earlier, we examined the relative abundances on the phylum and genus level. Over all analyzed libraries, 25 phyla were observed, from which the top six dominant phyla across all treatments in trout line A and B are Fusobacteriota, Firmicutes, Proteobacteria, Actinobacteriota, Desulfobacterota and Bacteroidota as shown in Fig. 4. Phyla with a presence of $<0.05$ were declared as 'Other'. Multiple contrast tests between treatments revealed, that intestinal microbiota from trout line $A$ exposed to stress is significantly dominated with Fusobacteriota $(p=0.04)$ compared to trout with no stress exposure. Contrary, increased abundance of Fusobacteriota in trout line B is associated with factor diet. In line B, trout which were not exposed to stress have significantly higher abundance of Fusobacteriota when fed with diet $\mathrm{V}$ in contrast to diet $\mathrm{F}(49 \%, \mathrm{p}=0.045)$ and further representing the maximum colonization of Fusobacteriota.

Abundance of Firmicutes is $>25 \%$ in all treatments, except for A-1-F (22\%), though not deviating significantly from the mean abundance and overall Firmicutes represents the most abundant phyla 
across all treatments. Proteobacteria is represented by $14-25 \%$ and $15-23 \%$ in trout line A and B, respectively. Less variation was observed in Actinobacteriota and Bacteroidota.

Prior to the actual trial (Supplements, Figure S3), initial sampling from breeding line A (A-init) show the highest abundance of Desulfobacterota (33\%) and lowest abundance of Firmicutes (12\%) compared to fish from experimental groups at the end of the trial. Initial sampling from breeding line B (B-init) represents the highest abundance of Firmicutes (50\%) compared to fish from experimental groups at the end of the trial and, thus, reducing the proportions of Fusobacteriota to the bottom of 1\%, albeit significantly lower than in treatment BOV $(p=0.0009)$. All samples of the experimental groups from breeding line $B$ showed a reduced abundance in Firmicutes compared to the initial sampling, although the only significant reduction was found in $B-1-V(p=0.037)$. In trout line $A$, abundance of Firmicutes was significantly higher in A-0-F, A-0-V and A-1-V $(p=0.039, p=0.0009, p=0.04)$ in contrast to A-init and Desulfobacterota were significantly decreased in all treatments in comparison to the start of the experiment. In trout line $B$, only fish from treatment groups not exposed to stress had significantly decreased abundances of Desulfobacterota in contrast to B-init (B-0-F: $p=0.03297$; B-0-V: $p=0.00453$ ).

Classification down to the genus level was achieved for 265 and 211 ASVs in line A and B, respectively. Taxa with an abundance of less than $0.01 \%$ were declared as 'Other' which led to 37 genera displayed in Fig. 5. The phylum Fusobacteriota is comprised of a single genera, Cetobacterium and hence close to absence in B-init. Thus, abundances from this phyla resemble the genus distribution. Stressed fish from tout line A showed significantly increased abundances in Bifidobacterium $(p=0.0249)$ and Enhydrobacter when fed with diet V. Otherwise, increased abundance of Staphylococcus $(p=0.0356)$ is observed in nonstressed fish when fed diet $\mathrm{V}$ in contrast to diet $\mathrm{F}$. In treatment $\mathrm{A} 1 \mathrm{~F}$, significantly reduced abundance of Lactobacillus compared to A-0-F was observed. Furthermore, Diet V significantly increased the appearance of Brevundimonas $(p=0.009)$, Candidatus Microhrix $(p=0.0229)$ and Blautia $(p=0.0418)$.

Evaluating abundance before and after the trial (Figure S4), significant increase of Cetobacterium in B-0-V was observed $(p=0.001)$ in contrast to initial sampling. Mycoplasma abundance was increased when diet $\mathrm{V}$ was fed, $A-0-\mathrm{V}(\mathrm{p}=0.00391)$ and $\mathrm{A}-1-\mathrm{V}(\mathrm{p}=0.00406)$. Microbes from the genus Bifidobacterium in trouts from line $B$ differentiated by higher abundance compared to intial samplings in $B-0-F(p=0.00525)$, $B-0-V(p=0.001)$ and $B-1-F(p=0.04310)$.

To determine which microbes contribute the most to the observed differences in microbial composition of intestinal gut contents, linear discriminant anylsis of effect size (LefSer) was conducted. Several taxa where identified as significantly differential abundant among treatments based on the estimated effect size. Out of this results, Table 2 shows the corresponding treatment and the LDA score for each significant genus. A prominent phylotyp, Cetobacterium from phylum Fusobacteriota, interestingly was significantly enriched in A-1-F and B-0-V. Most of the reads are classified as uncultured bacterium, while some could be assigned as Cetobacterium somerae. Furthermore, Mycoplasma (B-0-V), Photobacterium (A-1-F), Plesiomonas (B-0-V), Acinetobacter (B-0-F), Bifidobacterium (A-1-V) and Lactobacillus (A-0-F) were significantly enrichred in the respected treatments. 
Table 2

Linear discriminant analysis effect size (LEfSe) results of relevant features characterizing significantly $(\mathrm{p}<0.05)$ experimental treatments.

\begin{tabular}{|lllll|}
\hline treatment & Phylum & Genus & ef_Ida & padj \\
\hline A-0-F & Firmicutes & Lactobacillus & 4.357909 & 0.000369701 \\
\hline A-0-V & Bacteroidota & Bacteroides & 4.172772 & 0.034996576 \\
\hline A-0-V & Proteobacteria & Rhodobacteraceae_uncultured & 3.595482 & 0.011726509 \\
\hline A-1-F & Fusobacteriota & Cetobacterium & 4.892185 & 0.000596991 \\
\hline A-1-F & Proteobacteria & Photobacterium & 4.573118 & 0.000312278 \\
\hline A-1-F & Proteobacteria & Plesiomonas & 3.957476 & 0.008341385 \\
\hline A-1-F & Actinobacteriota & Dietzia & 3.757534 & 0.00177622 \\
\hline A-1-V & Actinobacteriota & Bifidobacterium & 4.414822 & 0.015852588 \\
\hline A-1-V & Actinobacteriota & Candidatus_Microthrix & 4.068949 & 0.032481135 \\
\hline B-0-F & Proteobacteria & Acinetobacter & 4.43116 & 0.018492112 \\
\hline B-0-F & Proteobacteria & Schlegelella & 3.893075 & 0.035253599 \\
\hline B-0-F & Proteobacteria & Roseomonas & 3.616095 & 0.018987449 \\
\hline B-0-V & Fusobacteriota & Cetobacterium & 4.878829 & 0.001335798 \\
\hline B-0-V & Firmicutes & Mycoplasma & 4.725006 & 0.037090428 \\
\hline B-0-V & Proteobacteria & Plesiomonas & 4.445059 & 0.000331659 \\
\hline B-0-V & Actinobacteriota & Rhodococcus & 3.720016 & 0.010631635 \\
\hline B-1-F & Actinobacteriota & Microbacteriaceae & 4.265236 & 0.026670446 \\
\hline B-1-F & Verrucomicrobiota & Luteolibacter & 3.480167 & 0.012180839 \\
\hline B-1-F & Proteobacteria & Bosea & 0.025550556 \\
\hline B-1-F & Proteobacteria & Rhizobiaceae & 0.0470602 \\
\hline B-1-F & Actinobacteriota & Rothia & 0.012180839 \\
\hline & & & 3.717075 & \\
\hline
\end{tabular}

\section{Discussion}

The present study is the first to investigate microbial community composition and performance of two rainbow trout (Oncorhynchus mykiss) breeding lines in a recirculating aquaculture system (RAS) exposed to handling stress while being fed with plant-based diets - important parameters in fish farming with regard to animal health and welfare. We believe, that this data is to shed more light in the commensal relationship of host and bacteria with a focus in this study on allochthonous inhabitants. Recently, the 
attention on the intestinal microbiome of aquaculture species has grown as it poses a key function to health, growth and disease status $[27,28]$. Consequently, investigating the interaction of factors that shape the microbiome such as diet, stress, environment or genetic state, among others, is crucial. By conducting marker gene sequencing at the hypervariable V3/V4 region of the 16S rRNA gene, of microbes residing in the intestinal gut material of rainbow trout haltered in a recirculating aquaculture system (RAS), modifications of the microbial landscape in the gut content of rainbow trout through interacting effects of handling stress and plant-based diets was evaluated. In this study, as fishmeal replacement (diet $\mathrm{V}$ ) we used $20.5 \%$ soy protein concentrate, $5 \%$ soybean meal and $15.2 \%$ wheat gluten. Otherwise, diet $F$ comprised of $6 \%$ soy bean concentrate, no soybean meal at all, $6 \%$ wheat gluten and $35 \%$ fishmeal. To simulate handling stress and thus trying to obtain its impact on microbial communities in combination with diet, fish were exposed to an external stress stimulus.

\section{Fish performance}

The experimental diets used in this trial were formulated according to commercial trout diets with varying inclusion levels of plant-based proteins and met all nutritive requirements for rainbow trout. Therefore, single effects of the experimental diets on the performance of fish were not expected and were not observed either. However, we hypothesized effects on performance parameters through the applied handling stressor and through an interaction of both factors (diet*stress), as we expected the microbiome to be a physiological modulator of the stress-response in fish [23]. Surprisingly, we also did not observe single effects of stress nor effects of a diet-stress-interaction in both breeding lines on fish performance, except for an increased daily feed intake (DFI) associated with diet V. Although statistically significant, the variance between the diets spreads between $\sim 0.03$ and is, thus, not biologically relevant.

\section{Alpha Diversity}

Mean microbial richness and diversity parameters did not significantly differ between treatment groups except for a gain in observed ASVs in stressed fish from trout line $B$, when fed diet $F$ in contrast to diet $V$. Indeed, samples of treatment groups B-0-F and B-0-V, have much fewer sequencing reads after filtering and decontamination procedure, since less than half of the samples are left in comparison to all other treatments. This might explain the statistical difference found in observed ASVs. However, the observed values in this study illustrate no particular results and are in the range of what has been observed in other microbiota studies in salmonids [3, 21, 29].

\section{Community composition of intestinal gut microbiota}

Analysis of the intestinal gut content at the phylum level revealed, that five taxa, namely Fusobacteriota, Firmicutes, Proteobacteria, Actinobacteriota and Bacteroidota, dominated the communities in both breeding lines and across all treatments. Our findings agree with previous studies which defined those phyla as major taxonomic groups of the vertebrate gastrointestinal tract [30] or yet more important in studies on rainbow trout $[3,31]$ or fish in general [32]. Exceptional is the presence of Desulfobacterota, which is not described in the previous mentioned 'core-microbiota' in fish. Previously described as 
Deltaproteobacteria, this anaerobic group represents sulfate-reducing and moreover fermentative syntrophic bacteria [33]. Highest abundance was detected in A-init and evaluating other treatments, this phyla can be classified as a low abundant phylum similar as in [31].

There seems to be no comprehensive interaction of diet and stress modulating the frequency of a particular phylum in both trout lines or in rainbow trout, respectively, when evaluating statistical results of relative abundance data (Supplementary table T5, T6). Although, bacteria from phylum Firmicutes display the dominant phyla in all treatments in both breeding lines, no significant statistical association with a certain diet or stress situation used in this study, like previous studies reported [10, 14, 32, 34, 35], could be made. Though not significant, a trend of increased Firmicutes occurrence could be observed in the primarily fish meal-based diet in trout line B. Another study analyzed stress stimuli like hypoxia or high fish density on the microbiome of the skin mucus layer in brook charrs and found a significant increase of pathogenic microbes and decrease of probiotic-like bacteria [36]. In the present study no such primary regulation of certain bacterial clades could be assign to the applied parameters possibly due to applicable intersample variation within the treatments. A further conclusion of previously mentioned studies is the increased abundance of Proteobacteriota in fish fed a fishmeal-based diet. In trout from line $\mathrm{B}$, though not significant, a similar effect can be observed. While the ratio of Firmicutes:Proteobacteria is a diet related effect, results of this study point to a considerable association of diet to the ratio of Fusobacteriota:Proteobacteriota. Even though there is no congruence in the abundance related to diet or stress in both trout lines, significant increase in treatment B-0-V makes the difference.

\section{Prevalence of peculiar genera in certain treatments}

Our analysis revealed, that the second most abundant phyla is Fusobacteriota. Interestingly, $99.97 \%$ of all ASVs belong to the genus Cetobacterium. Similar observations were made in omnivorous fish like common carp [37] with $93.94 \%$ coverage of Fusobacteriota and 8081 sequences out of 8085 of Fusobacteriota in a study by van Kessel et al. [38]. Furthermore, a diet-related study of largemouth bass revealed up to $89.9 \%$ coverage by Cetobacterium $[39,40]$. While in humans, Cetobacterium is associated with protein and carbohydrate fermentation [41], in freshwater fish Cetobacterium is known for the vitamin B12 production [42]. Those attributes correspond to the results elevated by aforementioned studies, that Cetobacterium abundance seems to be associated with an omnivorous - herbivorous diet in freshwater fish $[42,43]$. Presence of Cetobacterium in the gastrointestinal tract of rainbow trout was found to be the most abundant genus in the mucus layer of rainbow trout $[44,45]$ and yet another study claims an association with carnivorous fish [15]. While this study revealed a strong association with two certain treatments, marked presence of Cetobacterium was found in all gut material of sampled rainbow trout. Such general increased abundance of that species, leads to the assumption, that Cetobacterium holds a key role in digestion of certain nutrients and might be crucial for vitamin B12 metabolism in rainbow trout. Further studying in this context needs to be done to address the function of Cetobacterium within this microenvironment.

Another re-occurring bacterial genus is Mycoplasma, in this data allocated to the phylum Firmicutes, formerly classified as Tenericutes [46]. It has been reported, that Mycoplasma portrays a dominant 
representative commensal in the gastrointestinal tract of aquacultured rainbow trout [47] and other salmonids $[48,49]$ while found to be pathogenic in human hosts. Our findings support the hypothesis of Mycoplasma being a dominant microbe in the gut contents of salmonid hosts. While still lacking an exact detailed assertion of relationship and function between microbe and host, a long and close evolutionary and ecological commensal connection has been proposed. As salmonids are ammonotelic and are not able to de novo synthesize arginine (and its derivates), one explanation of the strong presence of Mycoplasma in general, may be due to its ability to detoxicate ammonia via its pathway by building up nitrogen carrier. While in the aforementioned studies the focus lied on metagenomic analysis of Mycoplasma, transcriptomic data of such scenarios may unravel the functional relationship between this microbe and the host. By the reason of frequently being reported in rainbow trout, the omnipresence of Mycoplasma in the gut contents is unequivocal, no matter what environmental conditions or diets being applied. Particularly, in the present treatments, no significant pattern between the conditions could be determined. Although, LEfSe results indicated substantive association of Mycoplasma with plantbased diet and no stress exposure in trout line $B$, a general connection between diet and stress cannot be related. However, a significant higher abundance than prior the trial could be related to the plant-based diet in both stress treatments, although this is only true for rainbow trout from line A.

\section{Stress as the main regulator of the microbiome}

The trout lines used in this study were raised in two distinct aquaculture breeding facilities and thus experienced high environmental variation. Because of the different life histories, conditions prior to the experiment, the different genotypes (genetic variation) and deviating adaptation parameters (time, feeding etc.), we expected a pre-determined bacterial community in each trout line due to early colonization events prior to the experiment. This hypothesis was supported by our findings, that the intestinal gut microbiota compositions from trout line $A$ and $B$ prior to the experiment clearly separated regarding to phylogenetic beta diversity analysis. Which of the multiple factors or a combination of all are responsible for these observations is not evident, but such microbial imprinting has been observed also in zebrafish [16] among others species. Therefore, we analyzed both breeding lines separately. At the end of the 59-day trial, the microbiome of both trout lines was significantly altered due to the experimental conditions in comparison to the initial timepoints of the respective trout line. Suggesting, that gut microbiome modification is affected by both, diet and stress in general, an early colonization can be resolved and supports the general idea of a flexible and malleable microbiome at several life stages [7, 9, 14]. Interestingly, the husbandry conditions in the RAS arranged to overcome the initial distinction by early colonization of both trout lines, suggesting an alignment of the microbial landscape due to the experimental set-up at the end of the experiment. This indicates, that life history effects on the microbial population can be modulated again. Nevertheless, there are differences between the two breeding lines in their response on the experimental treatments, suggesting a genetic impact on the stress-dietmicrobiome-interaction.

In trout line A, the microbiota was altered through an interaction of the factors diet and stress. Within each respective diet, the response of the intestinal gut microbiome was significantly affected by the 
stress level the fish was exposed to, which finally resulted in a significant discrimination of microbial composition based on phylogenetic distance matrices. As it has been proposed elsewhere, those results support the hypothesis of the connection between the physiological condition (induces by stress) and nutrition. As it has been shown in mammals [8] and e.g. zebrafish [23], the microbiome acts as part of the gut-brain-axis, a complex network, were both parts communicate with each other via several mechanisms. Neurotransmitter released through brain-controlled glands, induced by e.g. an external stressor, can be bound by microbes and thus change e.g. nutrient absorption or pathogen defense. Vice versa, members of the microbiota affect the host via afferent pathways of the vagus nerve to the central nervous system or by secreting metabolites that communicate to the immune systems or passing the intestinal barrier. Another study revealed [20], that temperature stress in combination with a soybean meal diet induces enteritis, a severe health issue in salmonid aquaculture. The question whether stress modulates the diet utilization or if a certain diet alters the coping with a stress stimulus cannot be answered up to this point. In trout line $B$, differences in the microbial gut composition are explained by the factor stress. Our results demonstrate, that the microbial gut composition of those trout is not affected by the diets we provided. Further investigation needs to be conducted, to elaborate what physiological changes in the host are triggered. While the stress in trout line $A$ is interacting with diet composition, trout line $B$ seems to be less affected by the diet which suggests, that the microbiota is able to maintain its microbial composition not depending on which diet was fed. Similar to a study on stress response of brook charr microbiome [50], the genetic background of rainbow trout, determines how susceptible the microbial composition is to stress and that is a trend we did observe in this study. Concluding, that the microbiome of rainbow trout fed with a plant-based diet are less susceptible to an external stressor in certain breeding lines.

\section{Conclusions}

Contemplating the experimental set-up of this study, the most influencing factor on the intestinal gut microbiota of rainbow trout, is the stress status. We found, that the response of the microbial composition to stress is presupposed by (i) the examined breeding line and (ii) which diet was provided. Based on those factors, the influence of stress on intestinal microbiota of rainbow trout changes. Although our data can demonstrate and corroborate shifts in the microbial community as a function of diet and/or stress, at this stage, we note no ability to predict how these shifts may impact the physiology of the fish or the microbiome, so far. Further research related to how a stress-diet constellation is affecting the mechanisms at a molecular level and especially the interaction of host and microbiome needs to be done. Furthermore, the data disclosed, that no effect of the aforementioned factors on alpha diversity or performance parameters was present. Additionally, the diet and stress constellation we provided had a significant impact on the abundance of two genera, namely Cetobacterium and Mycoplasma, who represent two recurrent genera within the intestinal gut microbiome of rainbow trout. What the exact role of those genera in such husbandry conditions is, needs further investigation. Since the results of the standard performance parameters revealed no indication of an influence by stress, diet 
or a diet-stress interaction, the gut microbiome composition, however, seems likely to be a sensitive biomarker for stress exposure in aquaculture.

\section{Material \& Methods}

\section{Animals \& experimental design}

The experiment was conducted at the "Gesellschaft für Marine Aquakultur mbH" (GMA, Büsum, Germany) (Fraunhofer Research Institution for Individualized and Cell-Based Medical Engineering (IMTE)). All experiments involving fish handling procedures were executed with the strict permission of the animal welfare officer of the GMA and the local authority of Schleswig-Holstein following the guidance of the German animal welfare law (TierSchG).

The multifactorial experiment was conducted as triplicates, composed of three parameters: breeding line $(\mathrm{A} \mid \mathrm{B})$, diet (F|V) and stress exposure (0|1).

Fish were reared in two Recirculating Aquaculture Systems (RAS), each consisting of $4.0 \mathrm{~m}^{3}$. Breeding lines were spatially separated in two RAS to avoid epidemic risk factors. Each RAS was subdivided into 20 tanks á $150 \mathrm{~L}$. A total of $2^{3}$ experimental groups were established in triplicates, e.g. 12 tanks were stocked with 18 individuals of breeding line $A$ and 12 tanks with 16 individuals of breeding line $B$, respectively, in order to assure a similar stocking density $\left(15 \mathrm{~kg} / \mathrm{m}^{3}\right)$. Tanks were stocked with female rainbow trout (Oncorhynchus mykiss) of either breeding line $\mathrm{A}$, obtained from Forellenzucht Trostadt (Forellenzucht Trostadt GbR, Tautenhahn, Germany) which originates from Troutlodge (Bonney Lak, USA) or breeding line B, purchased from Themar Fischzuchtanlage $\mathrm{GmbH}$ (Themar, Germany) originating from Frédéric Cachelou (Sarrance, France). Fish were exposed to constant environmental parameters comprised of $15.17^{\circ} \mathrm{C} \pm 0.44$ water temperature, $9.67 \mathrm{mg} \mathrm{I}^{-1} \mathrm{O}_{2}$ concentration, $\mathrm{pH} 7.2,4.62 \mathrm{ppt} \pm 0.63$ salinity, Ammonium $\left(\mathrm{NH}_{4}^{+}\right)$and nitrite $\left(\mathrm{NO}_{2}{ }^{-}\right)$which was checked on a daily routine. Light was provided for $15 \mathrm{~h}$ per day.

\section{Diets}

Rainbow trout were fed with two experimental diets formulated according to commercial trout diets differing in fish protein ( $35 \%$ diet F, $7 \%$ diet V; Table 3) and plant-based proteins ( $47 \%$ diet $F, 73 \%$ diet $\mathrm{V}$ ). Diets were manufactured by Sparos (Olhão, Portugal) and were isonitrogenous and isoenergetic. Two times per day $1.5 \%$ of the total biomass were fed per hand.

\section{Handling}

In order to simulate handling stress, fish were shooed with a fishing net twice a day for $60 \mathrm{sec}$. To avoid bias in feed intake, fish were stressed two hours after each feeding event. Stressed and non-stressed fish were spatially separated within the RAS (experimental block design) in order to avoid random effects in the non-stressed experimental groups caused by shooing in surrounding tanks. Prior to the trial, fish of breeding line A were acclimatized to the rearing system for 6 weeks, while fish of breeding line B 
acclimatized for 12 days due to logistic issues concerning fish acquisition. The experimental trial lasted 59 days in total. Fish performance parameters based on group measurements were acquired after 50 days in order to avoid additional handling stress effects for the final sampling. During adaptation, fish feeding rates were restricted to $0.5 \%$ of total biomass per day, applying commercial trout diets from BiomMar for line A and Skretting for line B, respectively. Before the start of the trial all fish stocked into the two RAS were individually weighed (initial body weight; IBW), as well as all sampled fish at the end of the trial for the microbiome analysis and some additional samples, used in another study for blood analysis, $(\mathrm{N}=240)$ after 59 days. Group-weights of experimental groups were determined on starting day and on days 15,35 and 50 to calculate specific growth rate (SGR), feed conversion ratio (FCR), daily feed intake (DFI), protein efficiency ratio (PER) and protein retention efficiency (PRE). 
Table 3

Experimental diets $\mathrm{F}$ (fishmeal-based) and $\mathrm{V}$ (plant-based). The Analytical components of the diet ingredients (\% of original substance) were provided by SPAROS Lda, Olhão, Portugal. Information about nutritional values and energy content were acquired at the GMA, Büsum.

\begin{tabular}{|lll|}
\hline Ingredient & F [\%] & V [\%] \\
\hline Fish meal LT70 & 35.00 & 7.00 \\
\hline Fish protein concentrate & 2.50 & 2.50 \\
\hline Soy protein concentrate & 6.00 & 20.50 \\
\hline Wheat gluten & 6.00 & 15.20 \\
\hline Corn gluten & 5.00 & 5.00 \\
\hline Soybean meal 48 & 0.00 & 5.00 \\
\hline Wheat meal & 15.00 & 11.40 \\
\hline Faba beans (los tannins) & 6.00 & 6.00 \\
\hline Fish oil & 13.92 & 14.64 \\
\hline Rapeseed oil & 9.28 & 9.76 \\
\hline Vitamin \& Mineral Premixtures INVIVO $1 \%$ & 1.00 & 1.00 \\
\hline Lutavit C35 & 0.05 & 0.05 \\
\hline Lutavit E50 & 0.05 & 0.05 \\
\hline Antioxidant & 0.20 & 0.20 \\
\hline Monocalcium phosphate & 26.50 & 26.48 \\
\hline L-lysine & 18.43 & 20.79 \\
\hline L-tryptophan & 0.00 & 1.00 \\
\hline DL-methionine & 0.00 & 0.10 \\
\hline Total & 0.00 & 0.10 \\
\hline Nutritional composition (as fed basis) & 0.00 & 0.50 \\
\hline Water content & 100.00 & 100.00 \\
\hline Ash content & & \\
\hline Protein content & 5.74 & 5.22 \\
\hline Fat content & 4.85 \\
\hline Carbohydrate content & 42.66 \\
\hline
\end{tabular}




\begin{tabular}{|lll|}
\hline Ingredient & F [\%] & V [\%] \\
\hline Gross Energy $\left[\mathrm{MJ} \mathrm{kg}^{-1}\right]$ & 23.54 & 23.92 \\
\hline
\end{tabular}

\section{Sample collection}

Before the start of the trial 21 fish from each breeding line and at the end of the trial (day 59) in total 169 fish ( $21 \mathrm{fish} /$ treatment, $7 \mathrm{fish} /$ tank) were randomly sampled for microbiome analysis as follows: animals were anesthetized with a blow on the head and killed afterwards by cutting the gill vein. By placing fish on clean surfaces, gastrointestinal tract was dissected using sterile scissors and scalpels. Feces were collected by squeezing out the entire intestinal tissue posterior the stomach towards the anus. Fecal samples were stored immediately on dry ice in $5 \mathrm{ml}$ sterile tubes. Blood samples of each fish for analysis of molecular stress markers were taken and results are being published elsewhere (unpublished data).

\section{DNA isolation, library preparation and sequencing}

To lyse bacterial cells, $250 \mathrm{mg}$ of homogenized gut content supplied with $1 \mathrm{ml}$ InhibitEx buffer in 0.70 mm Garnet Bead tubes was homogenized (SpeedMill PLUS, Analytik Jena GmbH, Jena, DE) for $45 \mathrm{sec}$. After an incubation time of $5 \mathrm{~min}$ at $550 \mathrm{rpm}$ by $95^{\circ} \mathrm{C}$, lysed cells were pelletized for $1 \mathrm{~min}$ at $20.000 \mathrm{rcf}$. Total bacterial DNA culled from fish gut contents, was subsequently extracted using $200 \mu \mathrm{l}$ supernatant as input for QIAmp® DNA fast stool mini kit (Qiagen, USA, Cat. no. 51604) which was conducted according to the manufacturer's instructions on a QIAcube ${ }^{\circledR}$ automation machine (Qiagen, USA). Additionally, 12 extraction blanks, to obtain possible contamination during DNA preparation, were included.

After verification of bacterial DNA presence via agarose (2\%) gel electrophoresis, the 16S rRNA gene V3V4 region was amplified using polymerase chain reaction (PCR) employed with uniquely barcoded forward and reverse primer (hV3F 'CCTACGGGAGGCAGCAG'; hV4R 'GGACTACHVGGGTWTCTAAT') [51] to combine all samples for a single NGS run. To amplify $16 \mathrm{~S}$ rRNA genes, a final volume of $26 \mu \mathrm{l}$ comprised of $5.0 \mu \mathrm{l} 5 \mathrm{X}$ Phusion HF buffer, $0.5 \mu \mathrm{l}$ dNTP $(10 \mathrm{mM}), 0.3 \mu \mathrm{l}$ Phusion Hot Start II Polymerase $(2 \mathrm{U} / \mu \mathrm{l}$; Thermo Fisher Scientific), $9.2 \mathrm{H}_{2} \mathrm{O}, 4 \mu \mathrm{l}$ of each primer $(100 \mu \mathrm{M})$ and $3 \mu \mathrm{l}$ microbial DNA template was prepared. Each PCR reaction plate included a negative control with nuclease free water to account for contamination and a reaction employed with a mock community (ZymoBIOMICS Microbial Community Standard) as a positive control composed of eight bacterial isolates with defined abundances, to verify adequate performance. Following an initial denaturation temperature of $98^{\circ} \mathrm{C}$ for $30 \mathrm{sec}, 30$ cycles were carried out as followed: $98^{\circ} \mathrm{C}(0: 09 \mathrm{~min}), 55^{\circ} \mathrm{C}(1: 00 \mathrm{~min})$ annealing temperature and $72^{\circ} \mathrm{C}(1: 30 \mathrm{~min})$ extension temperature. After cycling, amplificants were extended with a final round at $72^{\circ} \mathrm{C}$ for $10: 00 \mathrm{~min}$. Expected fragment size of 550 bp was verified via gel electrophoresis on a $2.0 \%$ agarose gel.

Sequencing of the V3-V4 region from the 16S rRNA gene amplicons of fish faeces, positive and negative controls (in total 294 sequencing samples) was executed at the Institute of Clinical Molecular Biology (IKMB, Kiel University) on an Illumina MiSeq platform (Illumina Inc., San Diego, CA, USA) using MiSeq Reagent kit v3 according to manufacturer's specification ending up with 2x300 bp paired-end reads. Raw 
sequences obtained during the study are stored at the NCBI Sequence Read Archive (SRA) and can be accessed via the SRA accession number SRP355371 or the BioProject ID PRJNA797926.

\section{Bioinformatics}

Demultiplexed reads, exempted from sequencing adapters, were provided by the sequencing facility as FASTQ-files of which read quality was examined using FastQC [52] and MultiQC [53]. FASTQ-files files were imported into Quantitative Insights Into Microbial Ecology 2 (Qiime2 2021.2.0 [54]) were the following processing was performed. The Cutadapt plugin [55] was used to trim any leftover primers and/or spacers. To denoise, filter for low quality reads and chimeras and to merge paired end reads, the integrated DADA2 plugin [56] was run with truncation parameters 270 for forward and 247 for reverse reads and a truncation quality cutoff of 5 . The outcome resulted in a feature table based on amplicon sequence variants (ASVs) and its corresponding representative sequences. Features with an occurrence in less than two samples or an overall frequency of less than 16 were discarded using the filter-features command of the feature-table plugin. To assign taxonomy to ASVs, a naïve Bayes classifier was trained against a SILVA 99\% database (release 138), with regions of interest extracted from full-length sequences using corresponding primer V3-V4 using the feature-classifier plug-in. Reads from the SILVA database were extracted to generate custom reference sequences. Features classified as Cyanobacteria, assumed to derive from plant chloroplast, and mitochondrial origin were excluded from further analysis. Only ASVs classified as Bacteria and identified at least to the phylum-level where kept. Based on extraction controls, five extraction batches were excluded from further analysis due to contamination effects. A phylogenetic tree was generated using a de novo approach by conducting a Multiple Alignment using Fast Fourier Transform (MAFFT) via the qiime phylogeny plugin. Mock community samples were separated from the data set and analyzed for accurate expected abundances according to the manufacturer.

The following analysis was performed using RStudio and the R package phyloseq [57]. For alpha diversity analysis, richness was estimated using observed features, Shannon diversity and Simpson index which additionally include evenness. All parameters were estimated based on the genus-level. To compare how many taxa are shared among samples (beta-diversity), unweighted - and weighted UniFrac distances were assessed using $\mathrm{R}$ package vegan [58] and visualized by non-metric multidimensional scaling (NMDS).

\section{Statistics}

Statistical evaluation of the data was executed using computation software R (2021). Growth performance parameter, Alpha diversity, relative abundances of taxa evaluation started with the definition of an appropriate statistical mixed model $[59,60]$. Both breeding lines were tested in separate models. The particular model included diet and stress, as well as all their interaction terms (two-fold) as fixed factors. The tanks were regarded as random factor. The residuals were assumed to be approximately normally distributed and to be heteroscedastic. These assumptions are based on a graphical residual analysis. Based on this model, a Pseudo $\mathrm{R}^{2}$ was calculated [61] and an analysis of variances (ANOVA) was conducted, followed by multiple contrast tests [62] in order to compare the several levels of the 
influence factors, respectively. The initial samples were tested against the treatments (sampling time) for alpha diversity and abundance data in a separate model while using the same parameters.

Multivariate analysis of beta diversity in rainbow trout gut microbiome communities was applied to weighted and unweighted UniFrac distances using adonis function from the vegan package with 999 permutation with diet and stress as interaction factor. Pairwise PERMANOVA were executed with pseudofactor treatment as factor and no p-value adjustment using the R package RVAideMemoire. Instead, required comparisons were chosen and p-values were adjusted manually using method 'holm'. Multivariate homogeneity of group dispersions (betadisper, vegan) using treatments as pseudofactor were conducted.

Linear discriminant analysis effect size (LEfSe) [63] was applied using the R package microbiomeMarker [64], to identify bacteria, which contribute the most to the differences in community by performing LDA scoring to estimate the effect size (threshold $\geq 3$ ) with treatment as pseudofactor.

\section{Declarations}

\section{Ethics approval and consent to participate}

The experiments conducted during this study were approved by the animal welfare officer of the "Gesellschaft für marine Aquakultur $\mathrm{mbH}$ " and the local authority of Schleswig-Holstein following the guidance of the German animal welfare law (TierSchG).

\section{Consent for publication}

Not applicable

\section{Availability of data and material}

Raw sequencing files are available from the NCBI Sequence Read Archive (SRA) via the Study ID SRP355371 within the BioProject PRJNA797926.

\section{Competing interests}

The authors declare that they have no competing interests.

\section{Funding}

The research conducted in this study was funded by the German Research Foundation (DFG) with the project ID 401653591: „Das Metatranskriptom der bakteriellen Darmgemeinschaft von Forellen in 


\section{Author contribution statement}

Conceptualization: $\mathrm{SCH}, \mathrm{CS}$

Formal analysis: MS, FTFG

Funding acquisition: $\mathrm{SCH}$

Investigation: $\mathrm{SCH}, \mathrm{FTFG}, \mathrm{MS}$

Methodology: MS, CB, HS

Project administration: $\mathrm{SCH}$

Resources: CS, AF

Supervision: $\mathrm{SCH}$

Visualization: MS

Writing - original draft: MS

Writing - review and editing: $\mathrm{SCH}$

\section{Supplementary Information}

Additional file 1, T1 to T6

Relative abundance data and statistical results from alpha and beta diversity (T1-T6)

Additional file 2, S1 to S5

Performance paramters and additional figures (S1 to S5)

\section{Acknowledgements}

The authors would like to thank the staff of the Fraunhofer Research Institution for Individualized and Cell-Based Medical Engineering (IMTE) (former Gesellschaft für Marine Aquakultur mbH, Büsum) for their support during the conduction of the feeding trials, as well as the staff of the microbiome lab of the Institute of Clinical Molecular Biology for their assistance during laboratory procedures. The authors would especially like to thank Mario Hasler (CAU) for his advice regarding the statistical analysis. The 
authors would also like to acknowledge the valuable support of Nils Peder Willassen and Terje Klemetsen (both UiT, Norway) during conceptualization of this study.

\section{References}

1. The State of World Fisheries and Aquaculture 2020. FAO. 2020.

2. Gatlin DM, Barrows FT, Brown P, Dabrowski K, Gaylord TG, Hardy RW et al. Expanding the utilization of sustainable plant products in aquafeeds: A review. Aquaculture Res. 2007;6:551-579.

3. Wong S, Waldrop T, Summerfelt S, Davidson J, Barrows F, Kenney PB et al. Aquacultured rainbow trout (Oncorhynchus mykiss) possess a large core intestinal microbiota that is resistant to variation in diet and rearing density. Appl Environ Microbiol. 2013;16:4974-4984.

4. Makkar HPS, Tran G, Heuzé V, and Ankers P. State-of-the-art on use of insects as animal feed. Animal Feed Science and Technology. 2014;38:1-33.

5. Naylor RL, Hardyb RW, Bureauc DP, Chiua A, Elliottd M, Farrelle AP et al. Correction for Ma et al., Selective activation of the M1 muscarinic acetylcholine receptor achieved by allosteric potentiation. Proceedings of the National Academy of Sciences. 2009;42:18040.

6. David LA, Maurice CF, Carmody RN, Gootenberg DB, Button JE, Wolfe BE et al. Diet rapidly and reproducibly alters the human gut microbiome. Nature. 2014;7484:559-563.

7. Michl SC, Ratten J-M, Beyer M, Hasler M, LaRoche J, and Schulz C. The malleable gut microbiome of juvenile rainbow trout (Oncorhynchus mykiss): Diet-dependent shifts of bacterial community structures. PLoS One. 2017;5:e0177735.

8. Foster JA, Rinaman L, and Cryan JF. Stress \& the gut-brain axis: Regulation by the microbiome. Neurobiol Stress. 2017:124-136.

9. Michl SC, Beyer M, Ratten J-M, Hasler M, LaRoche J, and Schulz C. A diet-change modulates the previously established bacterial gut community in juvenile brown trout (Salmo trutta). Sci Rep. 2019;9:2339.

10. Desai AR, Links MG, Collins SA, Mansfield GS, Drew MD, van Kessel AG et al. Effects of plant-based diets on the distal gut microbiome of rainbow trout (Oncorhynchus mykiss). Aquaculture. 2012:134142.

11. Araújo C, Muñoz-Atienza E, Nahuelquín Y, Poeta P, Igrejas G, Hernández PE et al. Inhibition of fish pathogens by the microbiota from rainbow trout (Oncorhynchus mykiss, Walbaum) and rearing environment. Anaerobe. 2015:7-14.

12. Askarian F, Zhou Z, Olsen RE, Sperstad S, and Ring $\varnothing$ E. Culturable autochthonous gut bacteria in Atlantic salmon (Salmo salar L.) fed diets with or without chitin. Characterization by 16S rRNA gene sequencing, ability to produce enzymes and in vitro growth inhibition of four fish pathogens. Aquaculture. 2012:1-8.

13. Ring $\varnothing$ E, Løvmo L, Kristiansen M, Bakken Y, Salinas I, Myklebust R et al. Lactic acid bacteria vs. pathogens in the gastrointestinal tract of fish: A review. Aquaculture Research. 2010;4:451-467. 
14. Ingerslev H-C, Gersdorff Jørgensen L von, Lenz Strube M, Larsen N, Dalsgaard I, Boye M et al. The development of the gut microbiota in rainbow trout (Oncorhynchus mykiss) is affected by first feeding and diet type. Aquaculture. 2014:24-34.

15. Liu H, Guo X, Gooneratne R, Lai R, Zeng C, Zhan F et al. The gut microbiome and degradation enzyme activity of wild freshwater fishes influenced by their trophic levels. Sci Rep. 2016:6:24340.

16. Roeselers G, Mittge EK, Stephens WZ, Parichy DM, Cavanaugh CM, Guillemin K et al. Evidence for a core gut microbiota in the zebrafish. ISME J. 2011;10:1595-1608.

17. Gajardo K, Rodiles A, Kortner TM, Krogdahl Å, Bakke AM, Merrifield DL et al. A high-resolution map of the gut microbiota in Atlantic salmon (Salmo salar): A basis for comparative gut microbial research. Sci Rep. 2016;6:30893.

18. Navarrete P, Magne F, Araneda C, Fuentes P, Barros L, Opazo R et al. PCR-TTGE analysis of 16S rRNA from rainbow trout (Oncorhynchus mykiss) gut microbiota reveals host-specific communities of active bacteria. PLoS One. 2012;2:e31335.

19. Perry WB, Lindsay E, Payne CJ, Brodie C, and Kazlauskaite R. The role of the gut microbiome in sustainable teleost aquaculture. Proc Biol Sci. 2020;1926:20200184.

20. URN PA, SCHRAMA JW, ROMBOUT JHWM, OBACH A, JENSEN L, KOPPE W et al. Soybean mealinduced enteritis in Atlantic salmon (Salmo salar L.) at different temperatures. Aquacult Nutr. 2008;4:324-330.

21. Brown RM, Wiens GD, and Salinas I. Analysis of the gut and gill microbiome of resistant and susceptible lines of rainbow trout (Oncorhynchus mykiss). Fish Shellfish Immunol. 2019:497-506.

22. Kelly $\mathrm{C}$ and Salinas I. Under Pressure: Interactions between Commensal Microbiota and the Teleost Immune System. Front Immunol. 2017:559.

23. Davis DJ, Bryda EC, Gillespie $\mathrm{CH}$, and Ericsson AC. Microbial modulation of behavior and stress responses in zebrafish larvae. Behav Brain Res. 2016:219-227.

24. Galley JD, Nelson MC, Yu Z, Dowd SE, Walter J, Kumar PS et al. Exposure to a social stressor disrupts the community structure of the colonic mucosa-associated microbiota. BMC Microbiol. 2014:189.

25. Partrick KA, Chassaing B, Beach LQ, McCann KE, Gewirtz AT, and Huhman KL. Acute and repeated exposure to social stress reduces gut microbiota diversity in Syrian hamsters. Behav Brain Res. 2018:39-48.

26. Uren Webster TM, Rodriguez-Barreto D, Consuegra S, and Garcia de Leaniz C. Cortisol-Related Signatures of Stress in the Fish Microbiome. Front Microbiol. 2020:1621.

27. Ingerslev H-C, Strube ML, Jørgensen LvG, Dalsgaard I, Boye M, and Madsen L. Diet type dictates the gut microbiota and the immune response against Yersinia ruckeri in rainbow trout (Oncorhynchus mykiss). Fish Shellfish Immunol. 2014;2:624-633.

28. Llewellyn MS, Boutin S, Hoseinifar SH, and Derome N. Teleost microbiomes: The state of the art in their characterization, manipulation and importance in aquaculture and fisheries. Front Microbiol. 2014:207. 
29. Dehler CE, Secombes CJ, and Martin SAM. Environmental and physiological factors shape the gut microbiota of Atlantic salmon parr (Salmo salar L.). Cutting Edge Science in Aquaculture 2015. 2017:149-157.

30. Colston TJ and Jackson CR. Microbiome evolution along divergent branches of the vertebrate tree of life: What is known and unknown. Mol Ecol. 2016;16:3776-3800.

31. Lyons PP, Turnbull JF, Dawson KA, and Crumlish M. Phylogenetic and functional characterization of the distal intestinal microbiome of rainbow trout Oncorhynchus mykiss from both farm and aquarium settings. J Appl Microbiol. 2017;2:347-363.

32. Ghanbari M, Kneifel W, and Domig KJ. A new view of the fish gut microbiome: Advances from nextgeneration sequencing. Aquaculture. 2015;Suppl. 5:464-475.

33. Murphy CL, Biggerstaff J, Eichhorn A, Ewing E, Shahan R, Soriano D et al. Genomic characterization of three novel Desulfobacterota classes expand the metabolic and phylogenetic diversity of the phylum. Environ Microbiol. 2021;8:4326-4343.

34. Rimoldi S, Terova G, Ascione C, Giannico R, and Brambilla F. Next generation sequencing for gut microbiome characterization in rainbow trout (Oncorhynchus mykiss) fed animal by-product meals as an alternative to fishmeal protein sources. PLoS One. 2018;3:e0193652.

35. Heikkinen J, Vielma J, Kemiläinen O, Tiirola M, Eskelinen P, Kiuru T et al. Effects of soybean meal based diet on growth performance, gut histopathology and intestinal microbiota of juvenile rainbow trout (Oncorhynchus mykiss). Aquaculture. 2006;1:259-268.

36. Boutin S, Bernatchez L, Audet C, and Derôme N. Network analysis highlights complex interactions between pathogen, host and commensal microbiota. PLoS One. 2013;12:e84772.

37. Li T, Long M, Gatesoupe F-J, Zhang Q, Li A, and Gong X. Comparative analysis of the intestinal bacterial communities in different species of carp by pyrosequencing. Microb Ecol. 2015;1:25-36.

38. van Kessel MA, Dutilh BE, Neveling K, Kwint MP, Veltman JA, Flik G et al. Pyrosequencing of $16 \mathrm{~S}$ rRNA gene amplicons to study the microbiota in the gastrointestinal tract of carp (Cyprinus carpio L.). AMB Expr. 2011;1:41.

39. He M, Li X, Poolsawat L, Guo Z, Yao W, Zhang C et al. Effects of fish meal replaced by fermented soybean meal on growth performance, intestinal histology and microbiota of largemouth bass (Micropterus salmoides). Aquacult Nutr. 2020;4:1058-1071.

40. Larsen AM, Mohammed HH, and Arias CR. Characterization of the gut microbiota of three commercially valuable warmwater fish species. J Appl Microbiol. 2014;6:1396-1404.

41. Finegold SM, Vaisanen M-L, Molitoris DR, Tomzynski TJ, Song Y, Liu C et al. Cetobacterium somerae sp. nov. from human feces and emended description of the genus Cetobacterium. Syst Appl Microbiol. 2003;2:177-181.

42. Tsuchiya C, Sakata T, and Sugita H. Novel ecological niche of Cetobacterium somerae, an anaerobic bacterium in the intestinal tracts of freshwater fish. Lett Appl Microbiol. 2007;0:071018031740002-??? 
43. Silva FCdP, Nicoli JR, Zambonino-Infante JL, Kaushik S, and Gatesoupe F-J. Influence of the diet on the microbial diversity of faecal and gastrointestinal contents in gilthead sea bream (Sparus aurata) and intestinal contents in goldfish (Carassius auratus). FEMS Microbiol Ecol. 2011;2:285-296.

44. Lyons PP, Turnbull JF, Dawson KA, and Crumlish M. Exploring the microbial diversity of the distal intestinal lumen and mucosa of farmed rainbow trout Oncorhynchus mykiss (Walbaum) using next generation sequencing (NGS). Aquac Res. 2017;1:77-91.

45. Gatesoupe FJ, Fauconneau B, Deborde C, Madji Hounoum B, Jacob D, Moing A et al. Intestinal microbiota in rainbow trout, Oncorhynchus mykiss, fed diets with different levels of fish-based and plant ingredients: A correlative approach with some plasma metabolites. Aquacult Nutr. 2018;5:1563-1576.

46. Parks DH, Chuvochina M, Waite DW, Rinke C, Skarshewski A, Chaumeil P-A et al. A standardized bacterial taxonomy based on genome phylogeny substantially revises the tree of life. Nat Biotechnol. 2018;10:996-1004.

47. Lyons PP, Turnbull JF, Dawson KA, and Crumlish M. Effects of low-level dietary microalgae supplementation on the distal intestinal microbiome of farmed rainbow trout Oncorhynchus mykiss (Walbaum). Aquac Res. 2017;5:2438-2452.

48. Rasmussen JA, Villumsen KR, Duchêne DA, Puetz LC, Delmont TO, Sveier H et al. Genome-resolved metagenomics suggests a mutualistic relationship between Mycoplasma and salmonid hosts. Commun Biol. 2021;1:579.

49. Cheaib B, Yang P, Kazlauskaite R, Lindsay E, Heys C, Dwyer T et al. Genome erosion and evidence for an intracellular niche - exploring the biology of mycoplasmas in Atlantic salmon. Aquaculture. 2021:736772.

50. Crespel A, Bernatchez L, Garant D, and Audet C. Quantitative genetic analysis of the physiological stress response in three strains of brook charr Salvelinus fontinalis and their hybrids. Journal of Fish Biology. 2011;7:2019-2033.

51. Klindworth A, Pruesse E, Schweer T, Peplies J, Quast C, Horn M et al. Evaluation of general 16S ribosomal RNA gene PCR primers for classical and next-generation sequencing-based diversity studies. Nucleic Acids Res. 2013;1:e1.

52. Andrews S. FastQC: A Quality Control Tool for High Throughput Sequence Data [Online].2010, http://www.bioinformatics.babraham.ac.uk/projects/fastqc/.

53. Ewels P, Magnusson M, Lundin S, and Käller M. MultiQC: Summarize analysis results for multiple tools and samples in a single report. Bioinformatics. 2016;19:3047-3048.

54. Bolyen E, Rideout JR, Dillon MR, Bokulich NA, Abnet CC, Al-Ghalith GA et al. Reproducible, interactive, scalable and extensible microbiome data science using QIIME 2. Nat Biotechnol. 2019;8:852-857.

55. Martin M. Cutadapt removes adapter sequences from high-throughput sequencing reads. EMBnet $j$. 2011;1:10.

56. Callahan BJ, McMurdie PJ, Rosen MJ, Han AW, Johnson AJA, and Holmes SP. DADA2: Highresolution sample inference from Illumina amplicon data. Nat Methods. 2016;7:581-583. 
57. McMurdie PJ and Holmes S. phyloseq: An R package for reproducible interactive analysis and graphics of microbiome census data. PLoS One. 2013;4:e61217.

58. Oksanen J, Blanchet FG, Friendly M, Kindt R, Legendre P, McGlinn D et al. Vegan: Community Ecology Package.2020, https://CRAN.R-project.org/package=vegan.

59. Pinheiro J, Bates D. Mixed-effects models in S and S-PLUS. Springer science \& business media. 2006.

60. Carroll RJ, Ruppert D. Transformation and Weighting in Regression. 1988.

61. Nakagawa S, Schielzeth H, and O'Hara RB. A general and simple method for obtaining R2 from generalized linear mixed-effects models. Methods Ecol Evol. 2013;2:133-142.

62. Hothorn T, Bretz F, and Westfall P. Simultaneous Inference in General Parametric Models. Biom. J. 2008;3:346-363.

63. Segata N, Izard J, Waldron L, Gevers D, Miropolsky L, Garrett WS et al. Metagenomic biomarker discovery and explanation. Genome Biol. 2011;6:R60.

64. Yang C. microbiomeMarker: microbiome biomarker analysis: MicrobiomeMarker 0.0.1.2021, https://github.com/yiluheihei/microbiomeMarker.

\section{Figures}




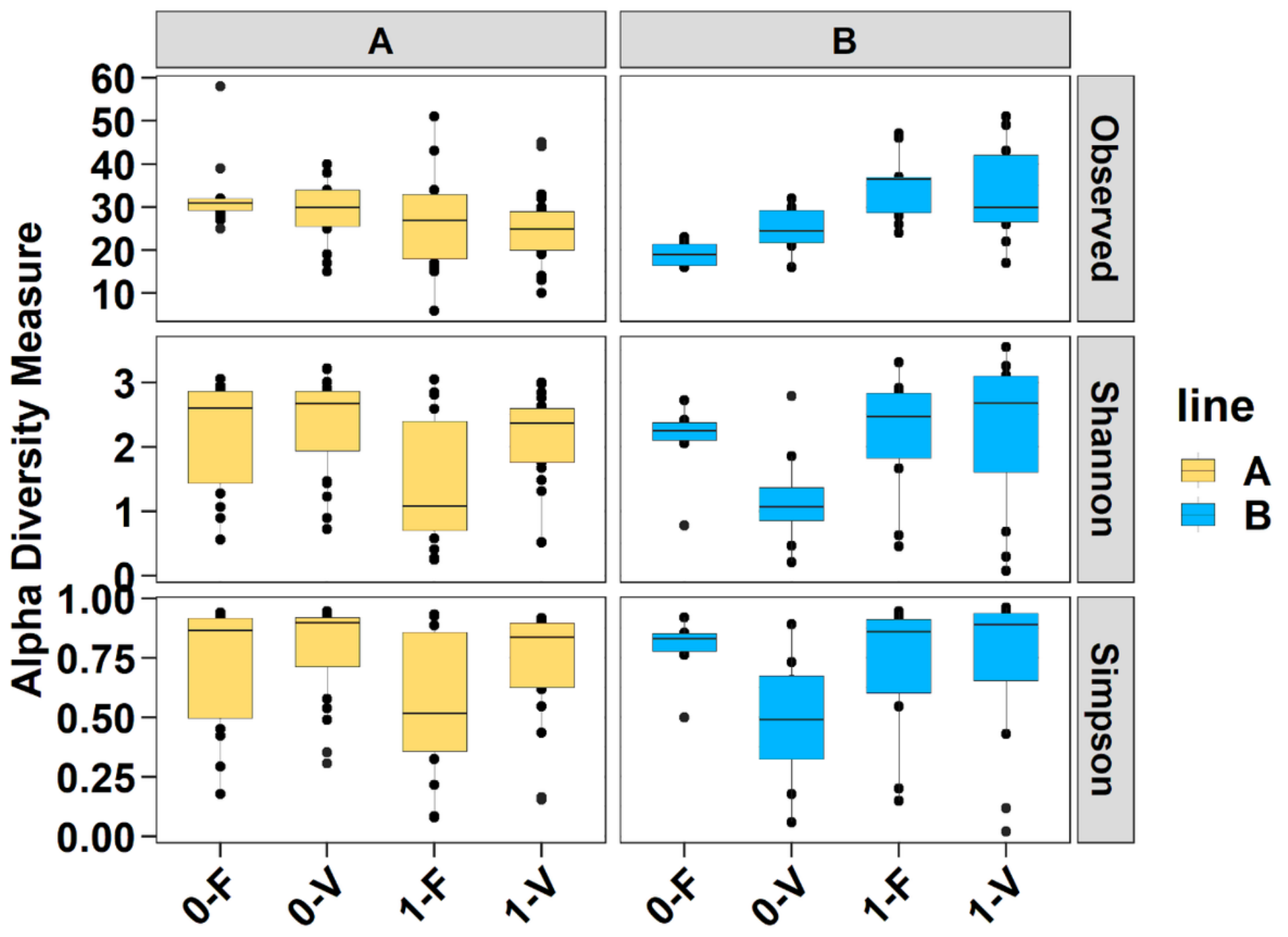

Figure 1

Alpha diversity indices including Observed ASVs, Shannon diversity and Simpson of the four treatments from trout line $A(A-0-F, A-0-V, A-1-F, A-1-V ;$ yellow) and B (B-0-F, B-0-V, B-1-F, B-1-V; blue). 

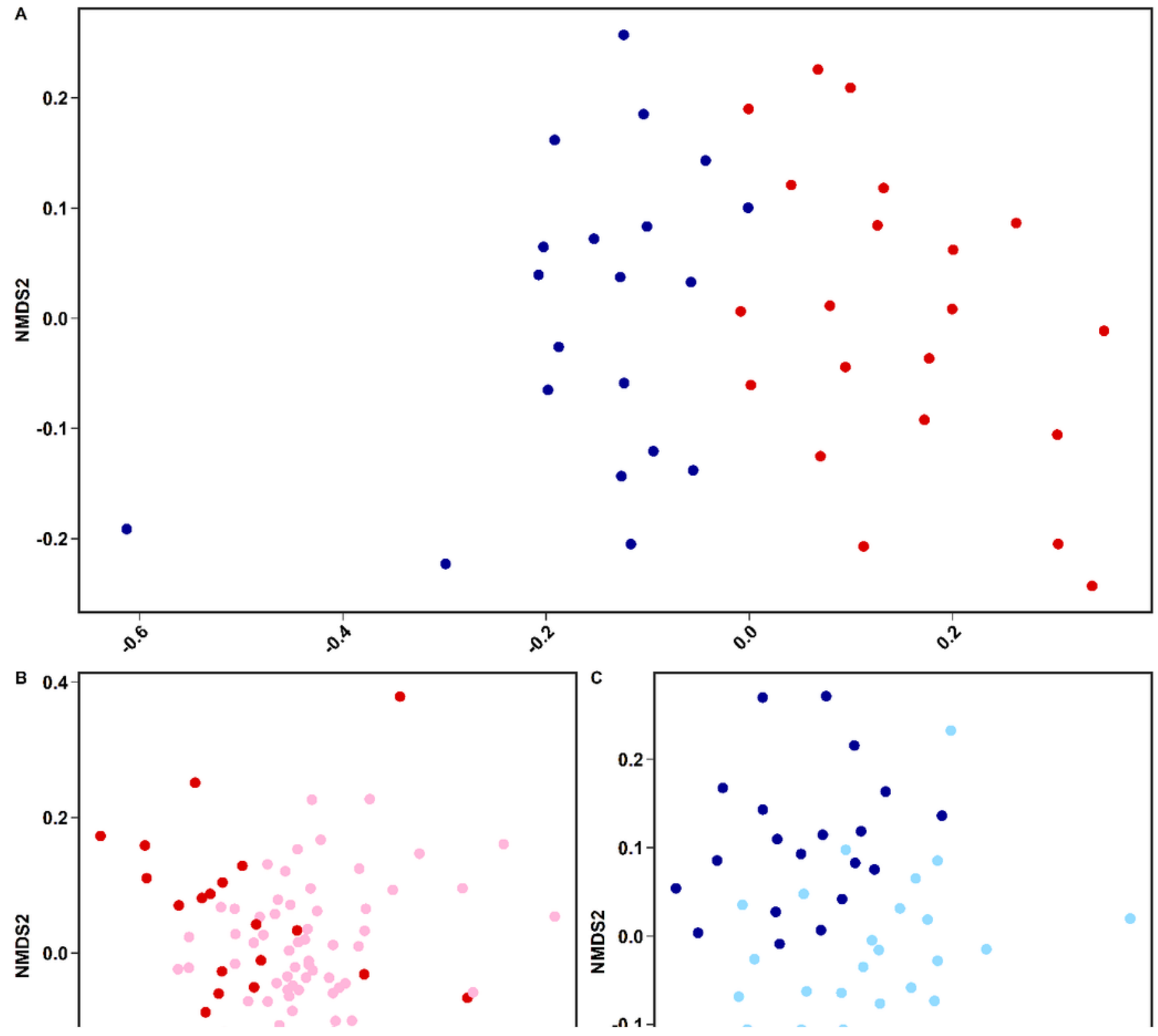

Figure 2

Microbial composition comparison among initial samples of both trout lines (A) and sampling time in trout line $A(B)$ and $B(C)$ on genus level data. NMDS on unweighted UniFrac distances. Initial samples are colored in dark red (A-init) and dark blue (B-init) and the corresponding samples after the treatment are light red for trout line $\mathrm{A}$ and light blue for trout line $\mathrm{B}$. 


\section{Figure 3}

Microbial composition comparison among treatments on genus level data. NMDS on unweighted UniFrac distances for trout line $A$ and $B(A, B)$ and weighted UniFrac distances for trout line $A$ and $B(C, D)$. Stress factor is colored with gold ( 0 ) and red (1) for trout line $A$ and green ( 0$)$ and blue (1) for trout line $B$. Diets are depicted with different shapes (F: circle, V: triangle).

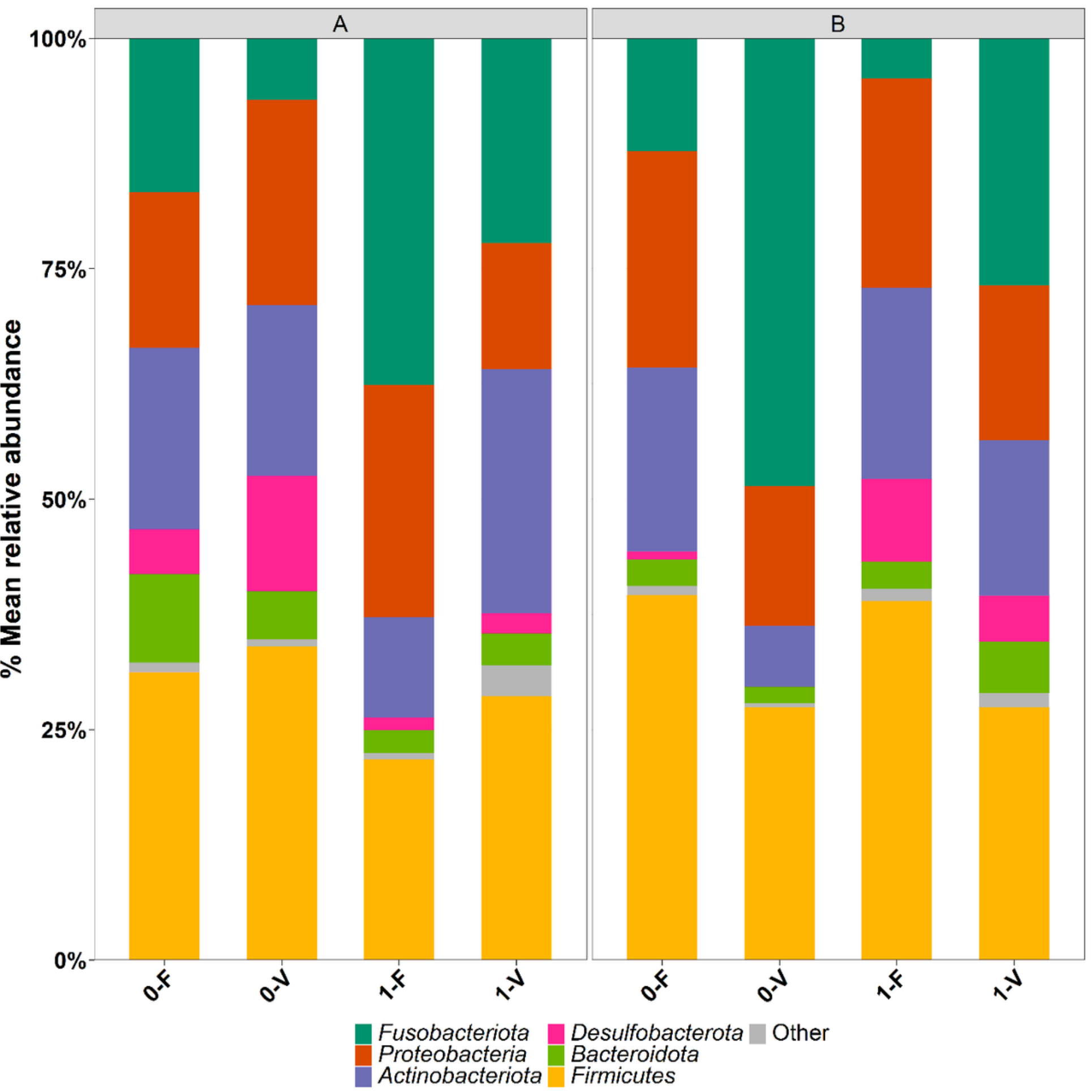




\section{Figure 4}

Mean relative abundance (\%) at the phylum level of the intestinal microbiota from trout line $A$ in the left and $B$ in the right panel. Each bar represents a diet-stress combination treatment within the trout lines. Phyla with a representation of $<0.05$ were pooled in category 'Other'. The arrangement of the bars is based on abundance, except for the most abundant phyla, which is placed at the bottom for legibility. The mean value joins data from each individual fish for the corresponding treatment across three modules.

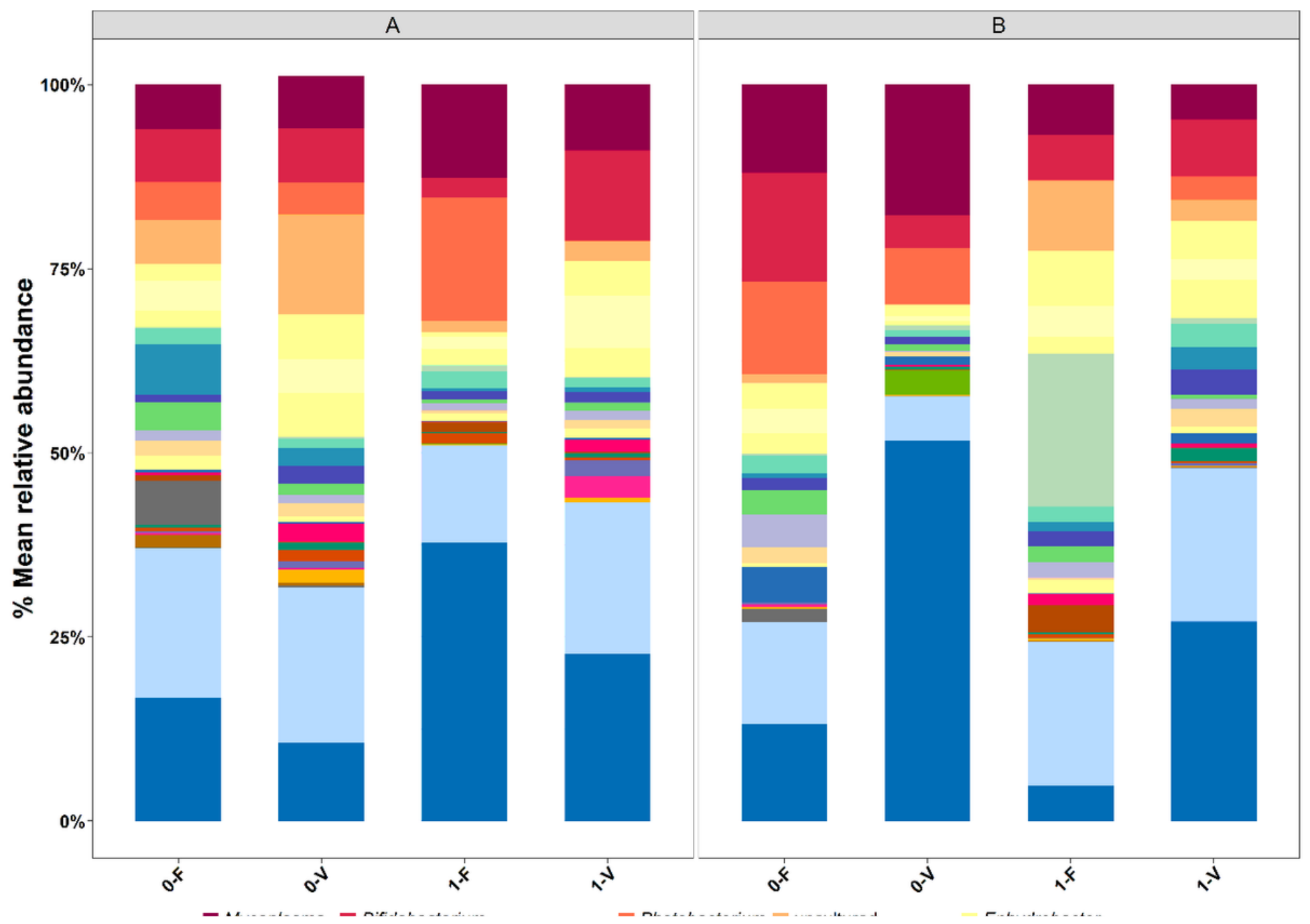

Figure 5

Mean relative abundance (\%) at the genus level of the intestinal microbiota from trout line A in left and B in the right panel. Each bar represents a diet * stress combination treatment within the trout lines. Genera with a representation of $1.5 \%$ were pooled in category 'Other'. The arrangement of the bars is based on abundance, except for the most abundant phyla, which is placed at the bottom for legibility. The mean value joins data on each individual fish for the corresponding treatment across three modules. 


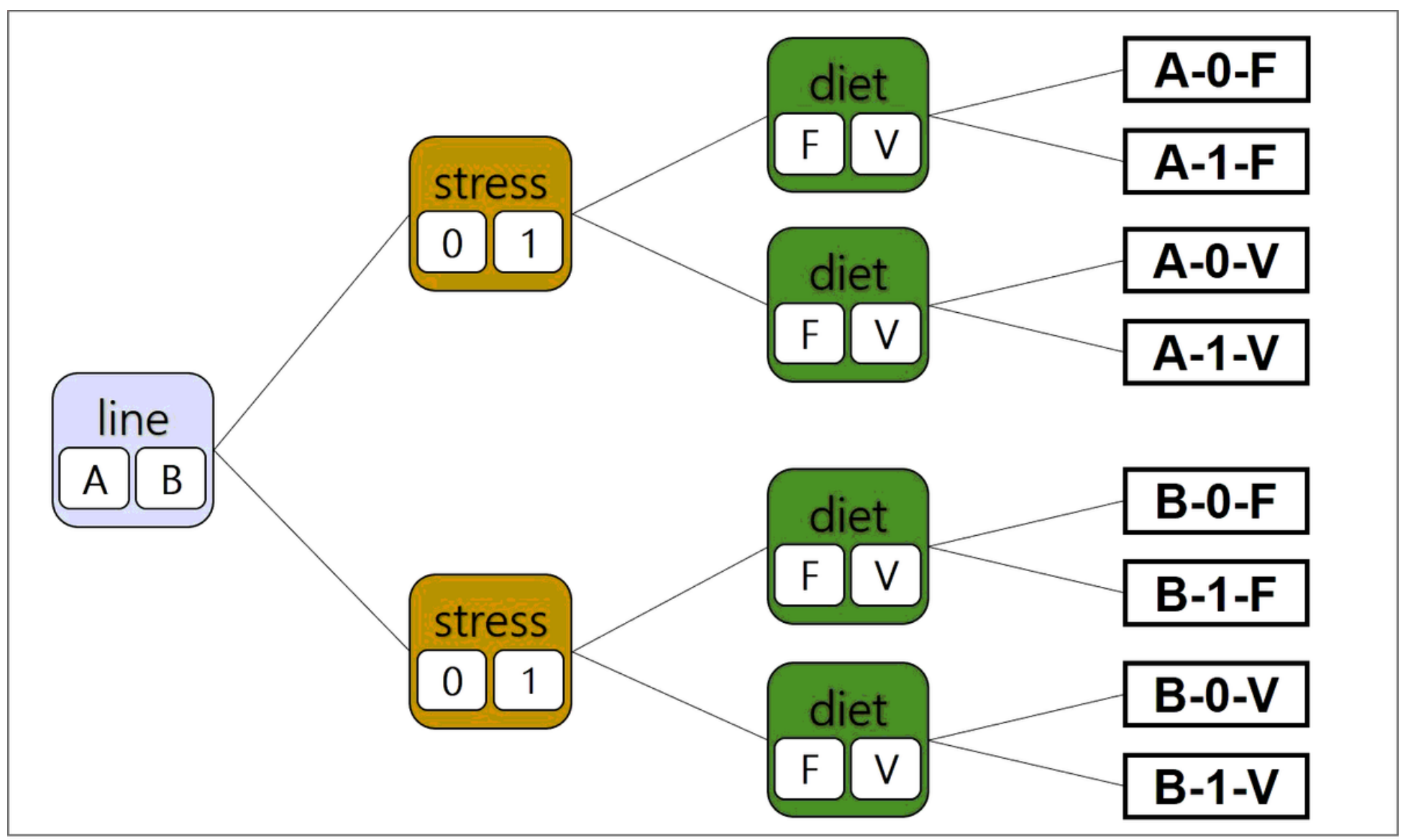

Figure 6

Scheme of treatments used in this study. Two different trout lines were used and identified as A or B. A 60 sec stress impulse twice a day, split the treatments in 1 for stressed and 0 for not stressed fish populations. Trout were fed either a fishmeal-based diet, shortened F or a plant-based diet V.

\section{Supplementary Files}

This is a list of supplementary files associated with this preprint. Click to download.

- 2022SUHRsupplementaryfigures.pdf 